\title{
MS005.P03
}

\section{Laboratory X-ray diffractometer for PDF experiments using Ag radiation}

$\underline{\text { Ripan Kumar Biswas }}^{1}$, Prosenjit Khan ${ }^{1}$, Anoop Kumar Mukhopadhyay ${ }^{1}$, Jiten Ghosh ${ }^{1}$, Muraleedharan Kuttanellore ${ }^{1}$

${ }^{1} A M M C D, C S I R$ - Central Glass And Ceramic Research Institute, Kolkata, India E-mail: ripanvc@gmail.com

The conventional crystallographic structure solution by X-ray Diffraction technique using Rietveld method prove its great potential for determination of the average structure of the materials for long range periodicity. Experimentally, the structural information of long range periodic atomic ordering of material is reflected in the Bragg's peaks while local or short range structure is reflected in the diffuse peaks. In order to obtain structural information about both average and local atomic structures, need a technique that will consider both Braggs peaks as well as diffuse peaks. Therefore, Total Scattering Atomic Pair Distribution Function (PDF) technique based on Debye Scattering function will be the only possible solution. At present synchrotron and neutron sources are the choice for PDF analysis for short range structure study. But there is a need for routine analysis of such type of samples in a conventional laboratory XRD system to get the quick feedback about the short range structure. PDF analysis can be performed in a Laboratory X-ray diffractometer using Ag radiation ( $\lambda=$ $0.5608 \AA$ ) to obtain maximum $Q$ value i.e. $22 \AA-1$. The present work will report PDF based methodology in a laboratory XRD system to extract structural information about nanostructured and disordered materials over short and long range for structural characterization of crystalline and amorphous materials.

Present work will report how this PDF technique used to unravel the structure of disordered materials and nanomaterials like amorphous silica, Ni, nano Ba-based Perovskite, etc for better understanding the materials at nano level. Structural information as obtained by the PDF analysis will help to control the performance of the disordered materials for tailoring the materials at nano scale. This method may be applicable to the characterization of the nanoscale crystalline and amorphous materials based on PDF analysis in Laboratory XRD system using Ag Radiation. This proposed experimental technique will help to quick feedback about local or disordered structure based on PDF using Ag radiation in a laboratory XRD system.

[1] Simon, J. L. Billinge. (2004). Z. Kristallogr. 219, 117-121

[2] Proffen, Th. et al. (2003). Z. Kristallogr. 218, 132-143

[3] Underneath the Bragg peaks: Structural Analysis of Complex Materials (2003). 1st ed., by T. Egami and S. J. L. Billinge, Elsevier Science B.V. Amsterdam
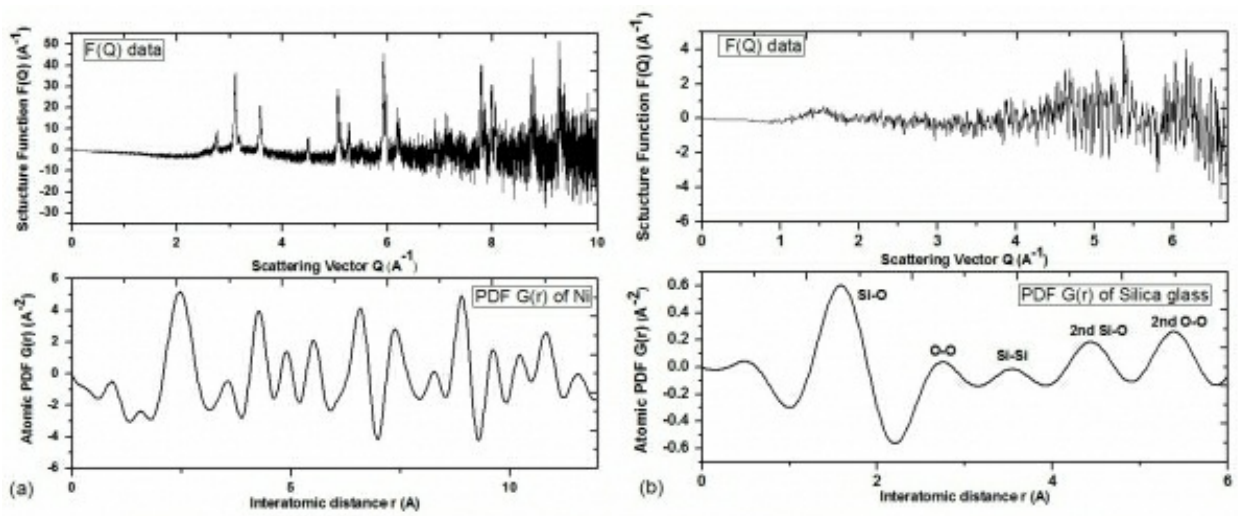

Fig.1. Reduced structure function $F(Q)$ and experimental PDF of (a) crystalline Ni and (b) amorphous Silica glass

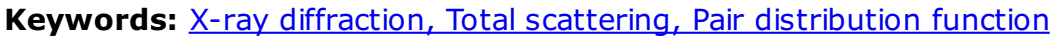

